\title{
ShRNA-mediated gene silencing of heat shock protein 70 inhibits human colon cancer growth
}

\author{
MING-AN ZHONG ${ }^{1 *}$, HUI ZHANG ${ }^{1 *}$, XIN-YONG QI ${ }^{2}$, AI-GUO LU ${ }^{1}$, TIAN-GENG YOU ${ }^{1}$, WEI GAO ${ }^{1}$, \\ XIN-LAI GUO ${ }^{1}$, ZHU-QING ZHOU ${ }^{1}$, YAO YANG ${ }^{1}$ and CONG-JUN WANG ${ }^{1}$ \\ ${ }^{1}$ Department of General Surgery, Shanghai East Hospital, School of Medicine, Tongji University; \\ ${ }^{2}$ Shanghai Animal Disease Control Center, Shanghai 200120, P.R. China
}

Received March 17, 2011; Accepted May 30, 2011

DOI: $10.3892 / \mathrm{mmr} .2011 .528$

\begin{abstract}
Heat shock protein 70 (HSP70), a chaperone involved in tumor progression, is overexpressed in various human tumors. However, its role in colon cancer progression is not completely understood. In the present study, two shRNA plasmid vectors against HSP70 were constructed and stably transfected into the colon cancer cell line HT29 to determine the effect of HSP70 on cell proliferation, cell cycle distribution and cell apoptosis in HT29 cells in vitro, and its effect on xenograft tumor growth and apoptosis in vivo. Cell proliferation was determined using MTT assay. The results revealed that HSP70 silencing efficiently inhibited the growth of HT29 cells in culture, induced cell cycle arrest at the G1 phase, and significantly increased apoptosis. Moreover, stable clones from the HSP70 shRNA-2 vector suppressed xenograft tumor growth and enhanced apoptosis in vivo compared with a mock and vector control group. In conclusion, specific HSP70 shRNA silencing may inhibit colon cancer growth, indicating that HSP70 silencing is a potential therapeutic strategy for the treatment of colon cancer.
\end{abstract}

\section{Introduction}

Colorectal carcinoma (CRC) is one of the most common malignant diseases in China. The incidence of colon cancer has been on the increase, becoming a major threat to public health. CRC is the third most frequently diagnosed cancer in the USA. In 2005, an estimated 105,950 new cases of colon cancer occurred. During the same year, an estimated 55,290 individuals succumbed to CRC (1). Therefore, it is vital that

Correspondence to: Dr Cong-Jun Wang, Department of General Surgery, Shanghai East Hospital, School of Medicine, Tongji University, 150 Jimo Road, Shanghai 200120, P.R. China

E-mail:wcj902@gmail.com

${ }^{*}$ Contributed equally

Key words: heat shock protein 70, RNA interference, apoptosis, colon cancer early detection and treatment be improved, particularly the efficacy of treatment to reduce mortality and extend the lives of patients.

Heat shock proteins (HSP), which are stress proteins acting as molecular chaperones, are generally induced by various environmental and pathophysiological stimuli (2). The protective function of HSP70 is related to its ability to promote folding of nascent polypeptides and to remove denatured proteins (3). However, HSP70 facilitates cancer cell survival and growth in various human tumor cells by inhibiting apoptosis and promoting proliferation $(4,5)$. The specific expression of HSP70 acting as an anti-apoptotic chaperone protein is commonly higher in cancer cells including breast, colon (6), ovarian (6) and pancreatic cancers (7). An elevated expression of HSP70 correlates with increased tumor cell proliferation, invasion, migration and poor prognosis. Therefore, HSP70 has been proposed as a putative target for cancer treatment.

RNA interference (RNAi), a genetic interference phenomenon that involves the post-transcriptional gene silencing mechanism, effectively suppresses the expression of a particular gene through the introduction of short interfering RNAs (8). However, the effect of down-regulated HSP70 expression by RNAi on colon cancer growth in vitro and in vivo has yet to be examined.

In this study, two shRNA plasmid vectors against HSP70 were constructed and transfected into the colon cancer cell line HT29 using RNAi technology, which persistently generated siRNA inside cells. Its effect on cell proliferation, cell cycle distribution and cell apoptosis in vitro as well as xenograft tumor growth and apoptosis in vivo were investigated.

\section{Materials and methods}

Construction of HSP70 shRNA-expressing plasmids. shRNA expression vectors were created with the use of a pSUPER.puro expression system. We synthesized two potential HSP70 gene (Genbank accession number: NM_005345)-specific target sequences (HSP70 shRNA-1: 5'-CACAAGAAGGACATCAGCC-3' and HSP70 shRNA-2: 5'-GGCCAACAAGATCACCATC-3'). For the negative controls, shRNA vectors were created using a scrambled sequence of the HSP70 target sequences (shRNA vector 
control: $\quad$ 5'-GCGTAGTCAACCACAACCA-3'). Using NIH BLAST analysis, sequences were confirmed to have no substantial homology to sequences in other vertebrate genes. Two annealed oligonucleotides, each encoding one of the target sequences followed by a 9-bp hairpin sequence (TTCAAGAGA) were ligated into the BglII and SalI sites of the pSUPER.puro plasmid to create the new recombinant plasmids, including the H1 RNA polymerase promoter and selective puromycin-resistant gene.

Cell culture and transfection. Human colon cancer cell line HT29 was maintained in RPMI-1640 (Gibco, USA), supplemented with $10 \%$ fetal bovine serum (Gibco), $100 \mathrm{u} / \mathrm{ml}$ penicillin $\mathrm{G}$, and $100 \mu \mathrm{g} / \mathrm{ml}$ streptomycin (Gibco) at $37^{\circ} \mathrm{C}$ in an atmosphere of $5 \% \mathrm{CO}_{2}$. Transient transfection of HSP70 shRNA and the vector control were carried out using Lipofectamine 2000 (Invitrogen, CA, USA). HT29 cells were trypsinized, plated on 96-well plates at a density of $1 \times 10^{4}$ cells/well 1 day prior to transfection and incubated for $24 \mathrm{~h}$. Transfection was performed according to the manufacturer's protocol. Cells were incubated for an additional 24, 48, 72 and $96 \mathrm{~h}$ post-transfection. For stable transfection, transfectants were selected using puromycin $(5 \mu \mathrm{g} / \mathrm{ml})$ for approximately 1 month.

Immunoblot analyses. Cells from mock (parental HT29 cells), shRNA vector control, HSP70 shRNA-1 and HSP70 shRNA-2 stable clones were lysed with RIPA lysis buffer. Equal amounts of protein were separated on $10 \%$ sodium dodecyl sulfate-polyacrylamide gel electrophoresis, transferred to polyvinylidene difluoride membranes (Amersham, Arlington Heights, IL, USA) by electroblotting and incubated with the antibodies anti-HSP70 and $\beta$-actin, (Santa Cruz Biotechnology, Santa Cruz, CA, USA). All antibodies were diluted in Tris-buffered saline and $0.1 \%$ (v/v) Tween-20 containing 5\% non-fat milk. Membranes were then incubated with horseradish peroxidase-conjugated secondary antibodies (Jackson ImmunoResearch, PA, USA). Protein bands were then developed with enhanced chemiluminescence detection reagents (Amersham Biosciences, Piscataway, NJ, USA).

Reverse transcription PCR. The relative expression levels of HSP70 mRNA in the human colon cancer cell line HT29 from mock, shRNA vector control, HSP70 shRNA-1 and HSP70 shRNA-2 stable clones were determined by reverse transcriptase-polymerase chain reaction (RT-PCR) analysis. The primers used were 5'-ACCAAGCAGACGCAGATCTTC-3' (sense), and 5'-TCGGCCAAGGTGTTGGCGTCC-3' (antisense). Total cellular RNAs were extracted from HT29 cells using TRIzol ${ }^{\mathrm{Tu}}$ reagent (Invitrogen) according to the manufacturer's protocol. For cDNA synthesis, $4 \mu \mathrm{g}$ of total RNA of each sample was used with random hexamer primers and a SuperScript ${ }^{\circledR}$ RT kit (Invitrogen). DNA was amplified under the following conditions: denaturation $94^{\circ} \mathrm{C}$ for $30 \mathrm{sec}$, annealing $53^{\circ} \mathrm{C}$ for $30 \mathrm{sec}$ and extension $72^{\circ} \mathrm{C}$ for $40 \mathrm{sec}$. HSP70 gene expression was normalized using reference primers for the $\beta$-actin gene: 5'-GGACTTCGAGCAAGAGATGG-3' (sense), and 5'-AGCACTGTGTTGGCGTACAG-3' (antisense). PCR products were separated in a $1.2 \%$ agarose gel and visualized by ethidium bromide staining.
Cell cycle distribution assay. Cells from mock, shRNA vector control and HSP70 shRNA-2 stable clones were harvested by trypsinization and centrifugation, washed twice with cold PBS, and fixed with $75 \%$ cold ethanol at $4^{\circ} \mathrm{C}$ overnight. The fixed cells were collected, washed twice with PBS and suspended in PBS containing $10 \mu \mathrm{g} / \mathrm{ml}$ propidium iodide (PI) (Sigma, St. Louis, MO, USA) and $100 \mu \mathrm{g} / \mathrm{ml}$ RNase A. The cells were then incubated at $4^{\circ} \mathrm{C}$ for at least $30 \mathrm{~min}$ in the dark to eliminate the intracellular RNA. Cell cycle distribution was determined using a flow cytometer.

Cell proliferation assay. The mock, shRNA vector control and HSP70 shRNA-2 stable clones were placed in 96-well plates at a density of $1 \times 10^{4}$ cells/well in $100 \mu 1$ medium, and incubated in a $37^{\circ} \mathrm{C}$ humidified incubator for attachment. MTT (Sigma) assay was performed according to the manufacturer's instructions. Briefly, cells were transfected with HSP70 vector control and HSP70 shRNA-2 after 24, 48, 72 and $96 \mathrm{~h}$. Then $20 \mu 1$ of $5 \mathrm{mg} / \mathrm{ml}$ MTT dissolved in PBS was added to each well, and cells were incubated for another $4 \mathrm{~h}$, followed by the addition of $150 \mu l$ dimethyl sulfoxide. The cells were left for $30 \mathrm{~min}$ at room temperature for colour development. Optical density was determined using a $490 \mathrm{~nm}$ filter on a microplate reader (Tecan, Groedig, Austria). The absorbance values were determined at days 1, 2, 3 and 4 in each group, respectively. Each assay was performed in triplicate and the experiment was repeated on at least three separate occasions.

Cell apoptosis detection. The mock, shRNA vector control and HSP70 shRNA-2 stable clones were trypsinized, collected, washed and then stained with Annexin V-FITC and PI (BD Bioscience) for $10 \mathrm{~min}$ at $4^{\circ} \mathrm{C}$ in the dark according to a standard protocol from BD Bioscience. Apoptotic cells were determined by flow cytometry (Becton Dickinson FACScan).

In vivo modeling. The nude mice (4-6 weeks old, $18-22 \mathrm{~g}$ of body weight, Animal Center of the Chinese Science Academy) were fed on a super-clean biological laminar flow shelf for 1 week. Mice were then randomly assigned to three groups $(n=5)$ and each HT29 clone was then inoculated into the mice. Briefly, $3 \times 10^{6}$ cells (mock, shRNA vector control or HSP70 shRNA-2 stable clones) were injected subcutaneously in $0.1 \mathrm{ml}$ of Hank's balanced salt solution into the right flank of each nude mouse. Tumor growth was monitored by measuring the perpendicular diameters with a caliper every 3 days, and calculating the tumor volume using the formula: $(\mathrm{V})=\mathrm{a} \times \mathrm{b} 2 \times 0.5$, in which $\mathrm{a}$ is the smallest and $\mathrm{b}$ the largest superficial diameter. After 3 weeks, the mice bearing the tumor xenografts were sacrificed and their tumor tissues were collected following an institution-approved protocol.

Immunohistochemistry staining. Mouse xenograft tumor tissues were harvested at the end of treatment, fixed in $10 \%$ neutral-buffered formalin, processed in paraffin and sectioned at $5 \mu \mathrm{m}$. Tissue sections were then subjected to immunohistochemical staining with antibodies against proliferating cell nuclear antigen (PCNA) according to the avidin-biotin complex procedure provided by the manufacturer (Vector). 


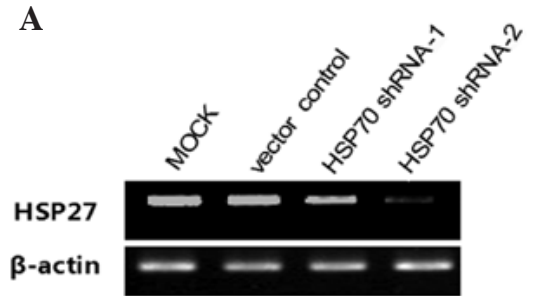

B

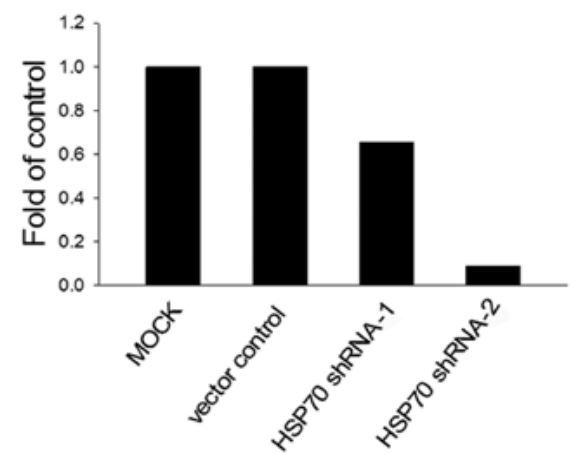

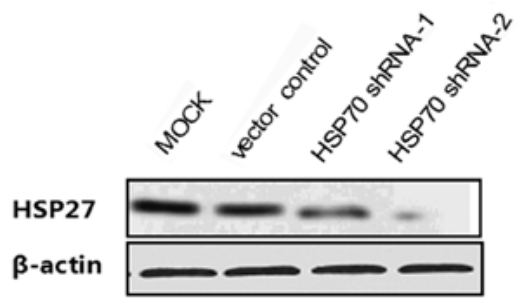

D

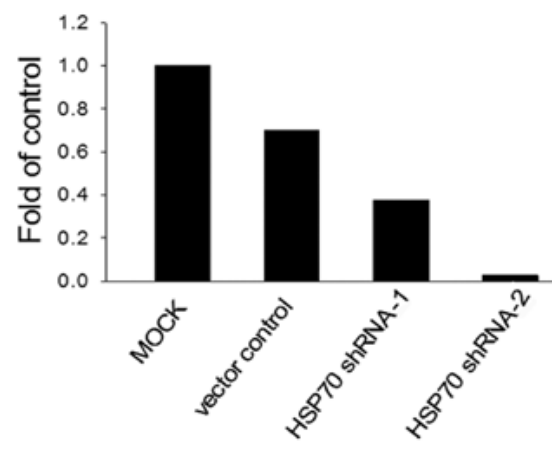

Figure 1. Stable silencing of HSP70 gene in HT29 cells. The pSUPER.puro plasmids containing two different HSP70 shRNA sequences were transfected into HT29 cells. Mock, parental cells; vector control, transfected with empty vector. Stable clones were selected in puromycin-containing medium. (A) The efficiency of HSP70 gene silencing was confirmed at mRNA levels by RT-PCR and (C) the protein levels by Western blot assays. (B and D) The optical density values of the corresponding bands were quantified by IPP 6.0 software (Media Cybernetics), as shown on the lower panel, and $\beta$-actin was used as an internal control.

Terminal deoxynucleotidyl transferase-mediated dutp nick end-labeling (TUNEL) assay. Formalin-fixed sections of tumor tissue $(5 \mu \mathrm{M})$ were collected from the mock, shRNA vector control and HSP70 shRNA-2 stable clone groups. The servers were then deparaffinized in xylene and hydrated in graded ethanol. The apoptotic cells were identified using a Cell Death Detection kit (Keygen, China) according to the manufacturer's instructions. Briefly, sections were incubated with $15 \mathrm{~g} / \mathrm{ml}$ proteinase $\mathrm{K}$ for $15 \mathrm{~min}$ at room temperature and washed with phosphate-buffered salinen (PBS). Endogenous peroxidase was inactivated by $3 \% \mathrm{H}_{2} \mathrm{O}_{2}$ for $5 \mathrm{~min}$ at room temperature and then washed with PBS. Sections were immersed in terminal deoxynucleotidyl transferase (TdT) buffer containing deoxynucleotidyl transferase and biotinylated dUTP in TdT buffer, incubated in a humidified atmosphere at $37^{\circ} \mathrm{C}$ for $90 \mathrm{~min}$, and then washed with PBS. The sections were incubated at room temperature for $30 \mathrm{~min}$ with anti-horseradish peroxidase-conjugated antibody, the signals were visualized with diaminobenzidine, and hematoxylin was used as a counterstain. We examined 4-5 random, x400 fields, from four slides per group.

Statistical analysis. The results were expressed as the mean \pm SD. The data were treated by ANOVA to determine statistically significant differences. $\mathrm{P}<0.05$ was considered to be statistically significant. Statistical analysis was performed using SPSS 13.0 software.

\section{Results}

HSP70 knockdown by shRNA-expressing vector. Two different siRNA sequences, as described in Materials and methods, were used to knockdown HSP70 gene expres- sion in HT29 cells. HSP70 shRNA-1 and HSP70 shRNA-2 markedly reduced HSP70 expression at both mRNA and protein levels compared to that of the mock and shRNA vector control groups. The inhibition rate of HSP70 shRNA-1 and HSP70 shRNA-2 were 34.4, 91 and 34, 90\%, respectively in HT29 cells compared with the mock and vector control groups at mRNA levels (Fig. 1A and B), and the inhibition rate of HSP70 shRNA-1 and HSP70 shRNA-2 was 62.4, 97.2 and 32.5, 57.3\%, respectively, in HT29 cells compared with the mock and vector control group at protein levels (Fig. 1C and D). However, shRNA-2 caused a marked reduction of HSP70 gene expression when compared to shRNA-1, reflecting a sequence-specific effect of siRNA (9). Taken together, these results indicated that HSP70 was overexpressed in human colon cancer cells, and transfection of the HT29 cells with the HSP70 shRNA plasmid strongly inhibited the expression of HSP70 in the cells.

Effect of HSP70 shRNA on cell proliferation. The HSP70 shRNA-2 stable clone was mainly used for further analysis due to its more marked reduction of HSP70 gene expression compared to shRNA-1, as mentioned above. HT29 cells were transfected with shRNA vector control or HSP70 shRNA-2 groups. Cell proliferation was examined by MTT assay for 1, 2, 3 and 4 days in each group, respectively. Results from the MTT assay indicated that relative cell proliferation in mock and shRNA vector control groups were gradually increased from day $1(1 \pm 0.06$ and $0.98 \pm 0.09)$ to day $4(2.40 \pm 0.087$ and $2.38 \pm 0.045$ ) in a time-dependent manner, but remained relatively unchanged in cells transfected with HSP70 shRNA-2 $(0.99 \pm 0.074)$. The statistical analysis revealed that the plasmid HSP70 shRNA-2 had significant inhibitive effects on the prolif- 
Table I. Cell cycle detected by flow cytometry (\%).

\begin{tabular}{lcccc}
\hline Cell & G0/G1 fraction & S fraction & G2/M fraction & S + G2/M \\
\hline Mock & $60.98 \pm 2.05$ & $19.67 \pm 0.94$ & $19.35 \pm 2.98$ & $39.02 \pm 2.05$ \\
Vector control & $63.38 \pm 1.74$ & $16.70 \pm 0.99$ & $19.92 \pm 2.19$ & $36.62 \pm 1.74$ \\
Hsp70 shRNA-2 & $79.19 \pm 2.10$ & $10.93 \pm 1.20$ & $9.88 \pm 2.43$ & $20.81 \pm 2.1^{\mathrm{a}, \mathrm{b}}$ \\
\hline
\end{tabular}

Cell cycle detected by flow cytometry. The G2 + S phase fraction of the HSP70 shRNA -2 group was the lowest compared with the mock and vector control groups. This result was consistent with that of the MTT assay. Data are expressed as the mean \pm SD of three experiments. ${ }^{\mathrm{a}} \mathrm{P}<0.001$ vs. mock, ${ }^{\mathrm{b}} \mathrm{P}<0.001$ vs. vector control group (ANOVA).

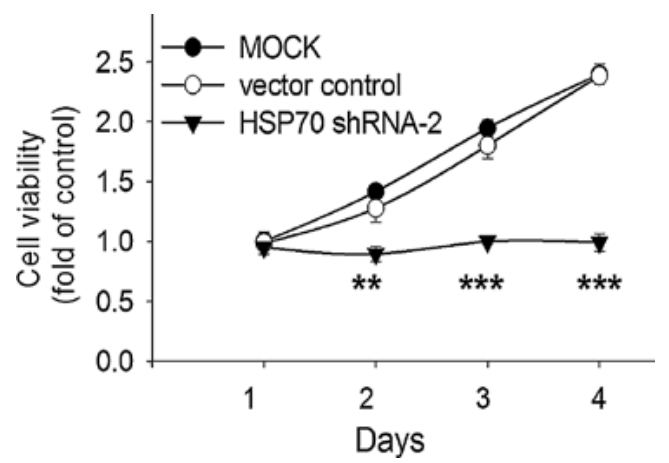

Figure 2. Effects on cell viability post-transfected with or without HSP70 shRNA. Mock, parental cells, vector control, transfected with empty vector and HSP70 shRNA-2 group. Cell viability was assessed using MTT to calculate the cell viability change (fold of control). Data are expressed as the mean $\pm \mathrm{SD}$, and ${ }^{* *} \mathrm{P}<0.01$ and ${ }^{* * *} \mathrm{P}<0.001$ (ANOVA).

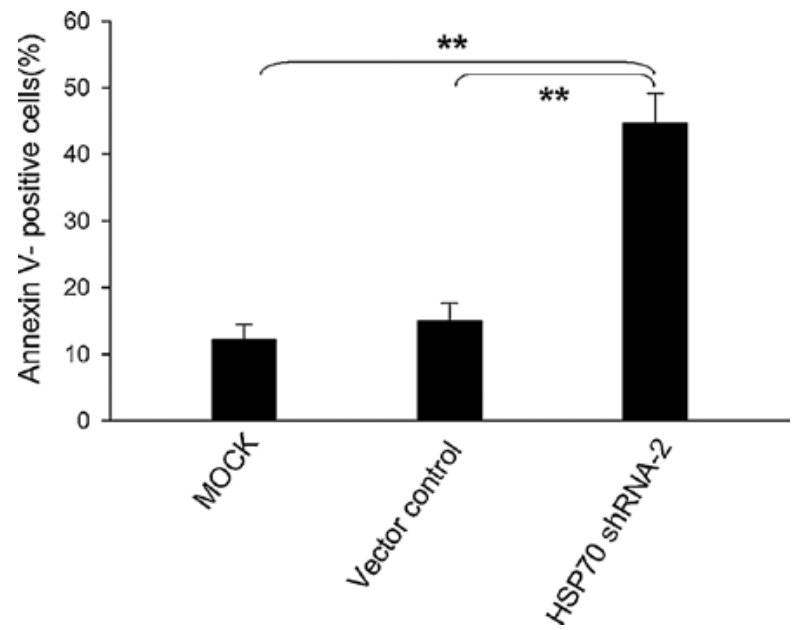

Figure 3. HT29 cells were stably transfected with the vector control and HSP70 shRNA- 2 construct. The percentage of apoptotic cells was measured using FITC-conjugated Annexin V antibody/PI staining by flow cytometry. Data are expressed as the mean $\pm \mathrm{SD}^{* * *} \mathrm{P}<0.01$ (ANOVA).

eration of HT29 cells compared with the mock and shRNA vector control groups, whereas the shRNA vector control group exhibited no significant inhibitive effect on cell proliferation compared to the mock group (Fig. 2).

Effect of HSP70 shRNA on cell cycle distribution and apoptosis in vitro. To investigate the effects of HSP70 shRNA on
A

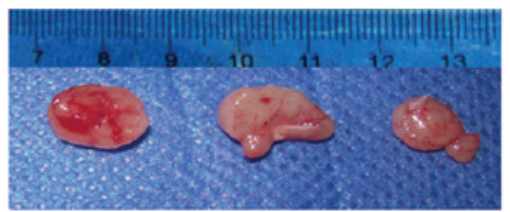

B

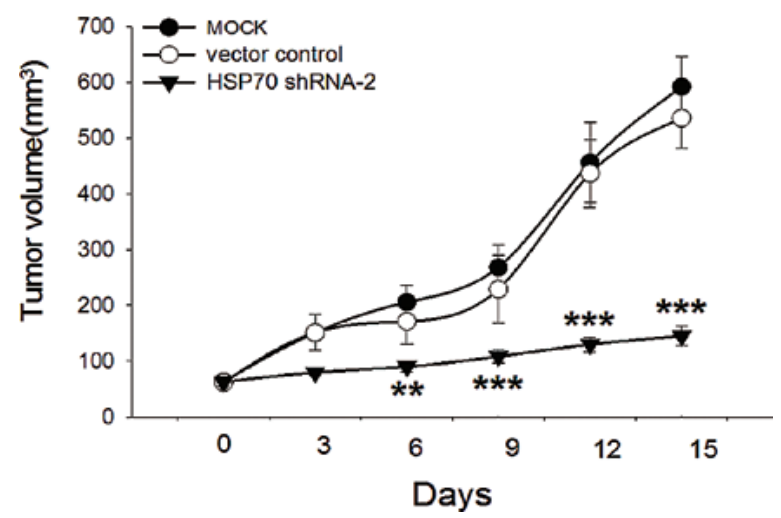

Figure 4. Effects of HSP70 shRNA-2 on colon cancer xenograft growth in vivo. (A) Tumor appearances in different groups. Mock (left panel), vector control group (middle panel), and HSP70 shRNA-2 group (right panel). The HSP70 shRNA-2 group tumor was significantly smaller than those in the two control groups. (B) Growth curves of different groups. The volume $\left(\mathrm{mm}^{3}\right)$ of tumors was monitored and recorded. Data are expressed as the mean $\pm \mathrm{SD}$ ${ }^{* *} \mathrm{P}<0.01$ and ${ }^{* * *} \mathrm{P}<0.001$ vs. vector control group (ANOVA).

the cell cycle, G0/G1, S and G2/M phase cells were detected by flow cytometry. HT29 cells were stably transfected with shRNA vector control or HSP70 shRNA-2 plasmid; cell proliferation was then detected by flow cytometry. In comparison to the mock and shRNA vector control groups, the shRNA-2 group exhibited cell accumulation in the G0/ G1 phase and reduction in the $\mathrm{S}$ and $\mathrm{G} 2 / \mathrm{M}$ phases. The total $S$ phase plus G2 phase fraction was greater than the mock and vector control groups. No statistically significant difference was observed between the mock and shRNA vector control groups (Table I). Furthermore, to investigate whether HSP70 shRNA-2 transfection increases apoptosis of human colon cancer cell line HT29, the apoptotic cell death was assessed using the Annenix V/FITC binding assay. Flow cytometry analysis also revealed the apoptotic rate of the mock, shRNA vector control and HSP70 shRNA-2 expressing cells were 


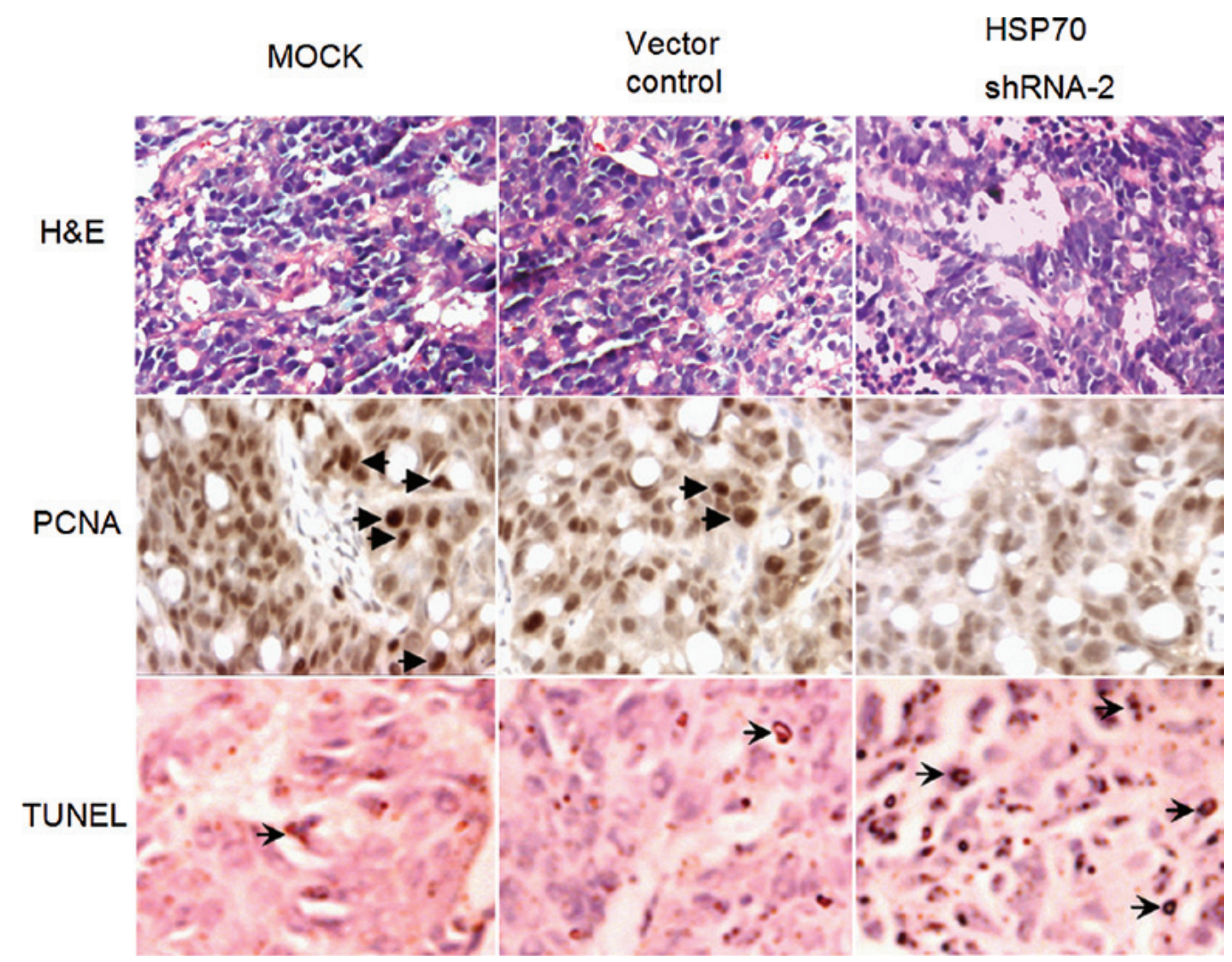

Figure 5. HSP70 shRNA-2 group displayed suppression of colon cancer growth and enhancement of apoptosis in vivo. Paraffin-embedded xenograft tumor sections in different groups were subjected for H\&E staining, immunohistochemical staining with anti-PCNA antibody and TUNEL agent staining. Tumor sections are shown. Under microscopy, large necrotic tissue was observed in HSP70 shRNA-2 tumors, while mock and vector control groups exhibited fine necrosis. Dark brown (arrowheads) shows strong positive immunostaining with anti-PCNA antibody. TUNEL-positive cells (apoptotic cells) are stained brown (arrows). There were more apoptotic cells in the samples from the HSP70 shRNA-2 group than in the mock and vector control groups.

$12.17 \pm 2.25,15 \pm 2.65$, and $44.67 \pm 4.5 \%$, respectively. The statistical analysis revealed a significant cell apoptotic increase in the HSP70 shRNA-2 group, compared to the mock and shRNA vector control groups. However, the apoptotic rates in the shRNA vector control-expressing cells were not significantly different from those in the mock group (Fig. 3).

Effect of HSP70 shRNA on colon cancer growth in vivo. To examine the effect of HSP70 silencing on tumor growth in vivo, we established xenografts in nude mice with the mock, shRNA vector control or HSP70 shRNA-2 stable clones. Tumor size was monitored every 2 days with a caliper. Growth of the xenografts derived from the shRNA vector control was similar to that in the mock group. However, the xenograft tumor volumes derived from the mock and shRNA vector control groups were markedly increased on day 7 following tumor cell inoculation as compared with the HSP70 shRNA-2 group, indicating that HSP70 silencing led to growth inhibition of the HT29 tumor cells in vivo (Fig. 4A and B).

HSP70 silencing inhibits cell proliferation and induces cell apoptosis. Hematoxylin and eosin (H\&E) staining carried out on formalin-fixed and paraffin-embedded tumor sections revealed that HSP70 shRNA-2 tumors were pale, with a large necrotic center (upper panel in Fig. 5). Tumor sections were subjected to immunohistochemical analysis of cellular markers for cell proliferation. Immunosignals for PCNA, a well-defined cell proliferation marker, were markedly reduced in HSP70 shRNA-2-derived tumors compared to the mock and vector control groups (middle panel in Fig. 5). Additionally, tumor sections were stained with the TUNEL agent to examine apoptotic cells. A small number of apoptotic cells were detected in tumors in the mock and vector control group, whereas a greater number of apoptotic cells were detected in the HSP70 shRNA-2 group (lower panel in Fig. 5).

\section{Discussion}

Numerous types of heat shock proteins are involved in the development of human colon cancer, including HSPs 10, 27, 70, 90 and 110 (10-13), of which HSP70 has attracted a great deal of attention as an anti-apoptotic chaperone protein. Numerous studies have revealed that the higher levels of HSP70 are associated with resistance to apoptosis, enhancement of cell growth and tumor development $(5-7,14)$.

Since HSP70 is crucial for the progression of various human tumors, HSP70 siRNA may become an effective therapeutic strategy for cancer. At present, various methods $(3,15,16)$ of targeting the HSP70 gene are available, such as indirect inactivation of HSP70 by inhibitors, and direct blocking of the functions of HSP70 by antisense oligonucleotides and RNAi. The latter is a potent tool in silencing specific genes in tumors due to its high performance, specificity, convenient manipulation and low toxicity. 
In the present study, human HSP70-specific shRNAs were designed as 64-mers containing a hairpin-loop flanked by HSP70 siRNAs and cloned into the pSUPER.puro vector. We selected a single clone in each group using $5 \mu \mathrm{g} / \mathrm{ml}$ puromycin approximately 1 month after transfection with HSP70 shRNAs (HSP70 shRNA-1 and shRNA-2). Results from RT-PCR and Western blotting showed that HSP70 mRNA and protein expression to be markedly down-regulated, subsequently resulting in cell viability decrease, cell cycle arrest and a cell apoptosis increase in vitro compared with the mock and vector control groups, indicating that HSP70 shRNA exhibited anti-proliferative and pro-apoptotic functions.

In addition, we observed that HT29 cells stably transfected with HSP70 siRNA grew slowly in nude mice as compared with the mock and vector control groups. These results were partially supported by a recent report that HSP70 depletion eradicated hepatocellular carcinoma (17), gastric cancer (3), cervical cancer (18) and bladder carcinoma (19) in mouse xenograft models. In the current study, the results from $\mathrm{H} \& \mathrm{E}$ staining, anti-PCNA immunohistochemical staining and the TUNEL assay obtained from these mice were consistent with in vitro results in HT29 cell lines, indicating that HSP70 shRNA possessed growth-inhibitory and apoptosis-enhancing effects on xenograft tumors in mice. It should be noted that this study has only focused on HSP70 silencing effects on cancer cell growth in vitro and in vivo. However, a variety of mechanisms, including the regulation of proliferation and apoptosis of closely related signal molecules could not be investigated. Therefore, further studies are required to examine the molecular mechanisms involved in HSP70 in cancer progression.

In conclusion, our experiments revealed that shRNA targeted against HSP70 was able to specifically mediate the HSP70 gene silencing, whose effect efficiently inhibited the growth of colon cancer cell line HT29 in vitro and in vivo. shRNA interference has potential as a treatment modality in human colon cancer.

\section{Acknowledgements}

This study was funded in part by the National Natural Science Foundation of China (30872510), the Shanghai Municipal Natural Science Foundation (no. 09ZR1416400) and the Natural Science Foundation of Shanghai (No. 064119620, 10411968400).

\section{References}

1 Jemal A, Murray T, Ward E, et al: Cancer statistics. CA Cancer J Clin 55: 10-30, 2005.
2 Ashburner $\mathrm{M}$ and Bonner JJ: The induction of gene activity in drosophila by heat shock. Cell 17: 241-254, 1979.

3 Xiang TX, Li Y, Jiang Z, et al: RNA interference-mediated silencing of the HSP70 gene inhibits human gastric cancer cell growth and induces apoptosis in vitro and in vivo. Tumori 94: 539-550, 2008

4 Yenari MA, Liu J, Zheng Z, Vexler ZS, Lee JE and Giffard RG: Antiapoptotic and anti-inflammatory mechanisms of heat-shock protein protection. Ann N Y Acad Sci 1053: 74-83, 2005.

5 Garrido C, Brunet M, Didelot C, Zermati Y, Schmitt E and Kroemer G: Heat shock proteins 27 and 70: anti-apoptotic proteins with tumorigenic properties. Cell Cycle 5: 2592-2601, 2006.

6 Katoh M, Koninkx J and Schumacher U: Heat shock protein expression in human tumours grown in severe combined immunodeficient mice. Cancer Lett 161: 113-120, 2000.

7 Aghdassi A, Phillips P, Dudeja V, et al: Heat shock protein 70 increases tumorigenicity and inhibits apoptosis in pancreatic adenocarcinoma. Cancer Res 67: 616-625, 2007.

8 Gartel AL and Kandel ES: RNA interference in cancer. Biomol Eng 23: 17-34, 2006.

9 Elbashir SM, Harborth J, Weber K and Tuschl T: Analysis of gene function in somatic mammalian cells using small interfering RNAs. Methods 26: 199-213, 2002.

10 Melle C, Bogumil R, Ernst G, Schimmel B, Bleul A and von Eggeling F: Detection and identification of heat shock protein 10 as a biomarker in colorectal cancer by protein profiling. Proteomics 6: 2600-2608, 2006.

11 Banerjea A, Ahmed S, Hands RE, et al: Colorectal cancers with microsatellite instability display mRNA expression signatures characteristic of increased immunogenicity. Mol Cancer 3: 21, 2004.

12 Cappello F, David S, Rappa F, et al: The expression of Hsp60 and Hsp10 in large bowel carcinomas with lymph node metastase. BMC Cancer 5: 139, 2005.

13 Tsuruta M, Nishibori $\mathrm{H}$, Hasegawa $\mathrm{H}$, et al: Heat shock protein 27 , a novel regulator of 5-fluorouracil resistance in colon cancer. Oncol Rep 20: 1165-1172, 2008.

14 Bausero MA, Page DT, Osinaga E and Asea A: Surface expression of Hsp25 and Hsp72 differentially regulates tumor growth and metastasis. Tumour Biol 25: 243-251, 2004.

15 Massey AJ, Williamson DS, Browne $\mathrm{H}$, et al: A novel, small molecule inhibitor of Hsc70/HSP70 potentiates Hsp90 inhibitor induced apoptosis in HCT116 colon carcinoma cells. Cancer Chemother Pharmacol 66: 535-545, 2010.

16 Zhao ZG and Shen WL: Heat shock protein 70 antisense oligonucleotide inhibits cell growth and induces apoptosis in human gastric cancer cell line sgc-7901. World J Gastroenterol 11: 73-78, 2005.

17 Zhu Q, Xu YM, Wang LF, et al: Heat shock protein 70 silencing enhances apoptosis inducing factor-mediated cell death in hepatocellular carcinoma hepg2 cells. Cancer Biol Ther 8: 792-798, 2009.

18 Garg M, Kanojia D, Saini S, et al: Germ cell-specific heat shock protein $70-2$ is expressed in cervical carcinoma and is involved in the growth, migration, and invasion of cervical cells. Cancer 116: 3785-3796, 2010.

19 Garg M, Kanojia D, Seth A, et al: Heat-shock protein 70-2 (HSP70-2) expression in bladder urothelial carcinoma is associated with tumour progression and promotes migration and invasion. Eur J Cancer 46: 207-215, 2010. 